\title{
Principal component and cluster analyses as supporting tools for co-crystals detection
}

\author{
Patrycja Saganowska $^{1} \cdot$ Marek Wesolowski $^{1}$
}

Received: 17 November 2016/Accepted: 28 April 2017/Published online: 6 May 2017

(C) The Author(s) 2017. This article is an open access publication

\begin{abstract}
The application of chemometry as a solution to a range of problems in the pharmaceutical industry has been the subject of increasing interest in recent years. Therefore, the objective of this work was to assess to what extent a chemometric approach based on principal component analysis (PCA) and cluster analysis (CA) can be used as a supplementary tool for co-crystal detection based on DSC, FTIR and Raman data. With this purpose in mind, potential co-crystals of furosemide with caffeine in molar ratios of $1: 1,2: 1$ and 1:2 were prepared using liquid-assisted grinding. The results of the study of furosemide and caffeine, their physical mixtures and potential co-crystals supported by PCA and CA revealed a significant discrepancy between the data obtained. In each case (DSC, FTIR and Raman study), a co-crystal in 1:1 molar ratio was detected and co-crystals in 2:1 and 1:2 molar ratios were also confirmed, by DSC, and FTIR and Raman, respectively. Detailed analysis of these data shows that the above discrepancies are connected with the sample features being measured. Thus, it was found that spectroscopic methods which reflect the chemical bonding of substances provide more reliable information on co-crystal formation than the DSC method which illustrates phase transitions. In conclusion, co-crystal formation could be only confirmed when the results obtained from PCA and CA examination of the data acquired from DSC, FTIR and Raman methods are compatible. It can therefore be affirmed that co-crystals of furosemide and caffeine were obtained only in a 1:1
\end{abstract}

Marek Wesolowski

marwes@gumed.edu.pl

1 Department of Analytical Chemistry, Medical University of Gdansk, Gen. J. Hallera 107, 80-416 Gdańsk, Poland molar ratio, while co-crystals in molar ratios of 2:1 and 1:2 were not formed.

Keywords Furosemide Caffeine $\cdot$ Co-crystals . Chemometry · Principal component analysis · Cluster analysis

\section{Introduction}

The poor solubility in water of active pharmaceutical ingredients (APIs) has become the most challenging issue to be overcome in the pharmaceutical industry, as the oral bioavailability of drugs is directly related to the solubility and dissolution rate of APIs [1]. Almost $40 \%$ of drugs used in health care contain APIs with low water solubility, and approximately $80 \%$ of potential APIs are poorly water soluble. Moreover, the poor water solubility of potential APIs is the main limiting factor in drug design and one that often precludes their introduction into medical practise, despite the desired pharmacological activity [2]. Therefore, different approaches towards improving this property have been applied, including, but not limited to, salt formation, particle size reduction, micellar solubilisation, solid dispersion and cyclodextrin complexation. Nonetheless, there is still a need to seek new methods of increasing API water solubility, since each of the above approaches has limited application [3]. Salt formation, for example, applies only to a small group of APIs which have ionisable groups; furthermore, the number of salt formers approved for use in the pharmaceutical industry is low [4]. It is worth mentioning at this point that enhancing API aqueous solubility frequently causes problems with drug instability under storage conditions. Against this background, co-crystals have recently gained great attention as a unique approach 
to improving solubility that could overcome some of the limitations of familiar methods.

In 2013, the US Food and Drug Administration (FDA) published guidance for the industry including classification of pharmaceutical co-crystals with particular emphasis on distinguishing co-crystals from other solid-state forms. According to FDA guidance, co-crystals are solid-state multicomponent materials which consist of at least two ingredients, API and neutral substance, called co-crystal former or coformer [5], which should be acceptable as pharmaceutical excipients and should have no toxic or adverse effects on the human body [6]. Components of cocrystals are bonded together in a crystal network via noncovalent week bonds without proton exchange, which allows co-crystals to be treated as a "drug-intermediate" and not as a new API. Co-crystallisation could be performed with APIs which do not have an ionisable group, in contrast to salt formation where the presence of the acidic or basic group is a prerequisite [7]. Hence, co-crystals present a great opportunity to enhance the low water solubility of those APIs lacking an ionisable group. The unique co-crystal lattice offers the possibility to modify the physicochemical properties of APIs. Co-crystallisation with a neutral component as coformer could alter the melting point of APIs, increase their physical and chemical stability, enhance solubility and consequently the bioavailability of APIs [8]. However, changes in API properties are often unpredictable and are dependent on the type of conformer used in co-crystallisation [6].

Many technological processes were used to prepare cocrystals, for instance dry-grinding, liquid-assisted grinding, the solvent-based method at ambient or elevated temperature, and co-crystallisation using supercritical fluid or ultrasound-assisted technology [9]. Of these techniques, grinding methods are employed for the screening of cocrystal formation, whereas solvent-based ones for scale-up and characterisation of co-crystals. Generally, three mechanisms, including molecular diffusion as well as intermediate formation of eutectic mixture or amorphous phase, are proposed for co-crystal formation by drygrinding [10]. However, the role of liquid addition in the most effective liquid-assisted grinding method is not yet fully understood. It may well act as a lubricant for the reaction or provide a medium to enhance molecular diffusion. Regardless of the method used in co-crystal preparation, its formation should be confirmed in each case by the correct tools such as differential scanning calorimetry (DSC) [2, 11], Fourier-transform infrared (FTIR) [2, 3] and Raman [10, 12] spectroscopies, powder X-ray diffraction (PXRD) [4, 13], and others [8, 14].

DSC can be used to measure a number of characteristic properties in a sample, such as fusion, crystallisation, glass transition, oxidation or stability [15]. The usefulness of
DSC is primarily due to the small quantity of sample applied to a study and the brief analysis time. Thus, it is used to detect co-crystal formation based mainly on alterations in melting points between API and the coformer in the co-crystallisation process. However, DSC has some limitations in co-crystal detection; for instance, a single endothermic event on a DSC scan may also indicate the melting of the eutectic mixture formed in the physical mixture. In this case, the construction of a binary phase diagram of the melting points of API and coformer mixed in different molar ratios allows co-crystals to be distinguished from eutectics [14]. Moreover, a single endothermic event could be also observed when the co-crystal and one of its components have similar melting points. In this situation, the broadening and shallowing of the peak without peak separation have been observed to obstruct DSC scan interpretation. As opposed to DSC, FTIR spectroscopy is a method which is not only focused on the behaviour of API and coformer in solid state but which reflects vibrational changes due to potential intermolecular interactions between components [16]. It can be used to detect co-crystals since a comparison of the FTIR spectra of physical mixtures with those of co-crystals allows structural differences to be recognised in the samples under study, such as formation of hydrogen bonding during cocrystallisation. Nevertheless, usage of FTIR spectroscopy in co-crystals detection is limited due to the presence of overlapping API and coformer bands in the co-crystals spectra, the disappearance of FTIR bands or the appearance of entirely new ones, as well as alterations in intensity and band broadening. Thus, whenever possible, supplementary methods should be employed.

Taking all the above into consideration, the aim of this study was to verify to what extent chemometry can be used as a supporting tool for detecting co-crystal formation. To this end, two unsupervised pattern recognition methods such as principal component analysis (PCA) and cluster analysis (CA) were used to gain information that can be indirectly acquired from DSC scans, and FTIR and Raman spectra. The aim of PCA is data reduction with the creation of a new set of variables called principal components (PCs) which are orthogonal to each other and describe variation in the data matrix [17]. CA, the second unsupervised approach was used as a continuation of PCA to assess whether a more sensitive discrimination of API physical mixtures with coformer and co-crystals can be expected when using a different classification algorithm. This method evaluates the clustering tendency of samples through an iterative process (agglomerative method) which associates the samples with relation to the distance between sample pairs and the difference in linkage algorithms according to which samples or clusters are merged [17, 18]. Our preliminary study revealed that PCA and CA make it 
possible to detect incompatibility in pharmaceutical mixtures based on thermal [19] and FTIR [20] data. Furthermore, Raman spectroscopy in conjunction with PCA enables us to gain a deeper insight into the transformation of physical mixture into co-crystal during dry-grinding [10] and PCA based on both PXRD and FTIR data is capable of differentiating polymorphic forms of carbamazepine and saccharin from co-crystals [21], while Raman spectroscopy combined with the partial least squares method permits the quantification of co-crystal and its coformer [22]. A strategy for the online monitoring of solvent-evaporation type co-crystallisation using near-infrared spectroscopy and PCA has also been developed [13, 23-25].

Co-crystal of furosemide as an API with caffeine (Fig. 1) as a coformer was chosen as the model substance for the study. The co-crystal was prepared using liquid-assisted grinding with furosemide to a coformer molar ratio of 1:1. Its chemical structure and physicochemical properties have been studied in more detail elsewhere. For instance, singlecrystal and powder X-ray diffraction revealed that cocrystal unit consists of one molecule of furosemide and one of caffeine bonded together via hydrogen bonds [2]. PXRD patterns were also used for confirming physical stability of furosemide-caffeine co-crystals before and after wet milling [25]. Furthermore, PXRD and DSC proved that furosemide-caffeine co-crystals formed a eutectic mixture with excess of caffeine, whereas no such mixture was formed with excess of furosemide [26].

\section{Materials and methods}

\section{Chemicals}

Furosemide and caffeine were purchased from SigmaAldrich, St. Louis, USA. The purity of both substances was $\geq 98 \%$. Acetonitrile was obtained from J.T. Baker, Gliwice, Poland. The purity of solvent was $\geq 99.8 \%$.

\section{Sample preparation}

Binary physical mixtures of furosemide with caffeine in molar ratios 1:1, 2:1 and 1:2 were obtained by gentle
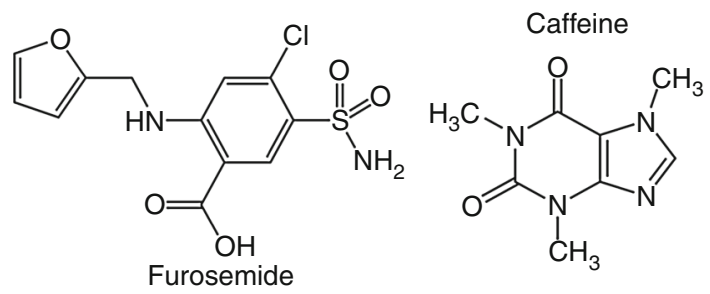

Fig. 1 Chemical formulas of furosemide and caffeine mixing of both components in agate mortal for $5 \mathrm{~min}$. Cocrystals were prepared using liquid-assisted grinding method. Adequate quantities of furosemide and caffeine in molar ratios $1: 1 ; 2: 1$ and 2:1 were mixed in agate mortar for $20 \mathrm{~min}$ using pestle after adding 5-6 drops of acetonitrile.

\section{Differential scanning calorimetry (DSC)}

DSC scans of furosemide, caffeine, mixtures of both components and their co-crystals were obtained using a heat-flux DSC instrument (model $822^{\mathrm{e}}$, Mettler Toledo, Schwerzenbach, Switzerland) coupled with a STARe software. Roughly $4 \mathrm{mg}$ of a sample was put into $40-\mu \mathrm{L}$ flat-bottomed aluminium pans closed with perforated lids. Measurements were carried out over the range of 25-300 ${ }^{\circ} \mathrm{C}$ at a heating rate of $10{ }^{\circ} \mathrm{C} \mathrm{min}^{-1}$ under nitrogen stream at a flux rate of $70 \mathrm{~mL} \mathrm{~min}{ }^{-1}$.

\section{FTIR spectroscopy}

A Nicolet 380 FTIR spectrometer (Thermo Fischer Scientific, Madison, USA) with a DTGS KBr detector and an OMNIC software was exerted to collect IR spectra of the samples. Hydraulic press (Specac, Orpington, UK) was used to prepare pellets for IR analysis. Each of pellets contained $1 \mathrm{mg}$ of a sample and $100 \mathrm{mg}$ of $\mathrm{KBr}$ (Merck, Darmstadt, Germany). Measurements were carried out over the spectral range of $4000-400 \mathrm{~cm}^{-1}$ with resolution of $4 \mathrm{~cm}^{-1}$. Before each measurement, background spectra were taken with average 16 scans.

\section{Raman spectroscopy}

Raman spectra were recorded on a DXR SmartRaman spectrometer (Thermo Fisher Scientific, Madison, USA). The spectrometer was equipped with a $15-\mathrm{mW}$ DXR $780 \mathrm{~nm}$ laser with a slit width of $25 \mu \mathrm{m}$, Raleigh filter, CCD detector and an OMNIC software. The measurements were run over the range of 3413-99 $\mathrm{cm}^{-1}$. Exposure time was $1 \mathrm{~s}$ (twice).

\section{Data analysis}

DSC scans, and FTIR and Raman spectra were interpreted with the aid of PCA and CA. The data acquired from Raman and FTIR spectra were pre-processed with a standard normal variate algorithm. In all matrices, the number of rows including physical mixtures and co-crystals prepared in molar ratios of 1:1, 1:2 and 2:1 was constant at a figure of six, whereas the number of variables differed for each matrix. The matrix of the data constructed for DSC scans included heat flow values acquired every $3{ }^{\circ} \mathrm{C}(98$ 
variables). The transmittance values of furosemide and caffeine at characteristic spectral ranges (2930-3400 and $821-1860 \mathrm{~cm}^{-1}$ ) were used to create an FTIR spectra matrix consisting of 51 columns. The matrix of the data acquired from Raman spectra included 20 variables reflecting Raman intensity values at selected wavenumbers of Raman shifts characteristic for furosemide and caffeine. PCA and CA calculations were carried out using Statistica 10 software (StatSoft Inc., Tulsa, USA), and CA performed to allow for the Euclidean distance between pairs of samples and Ward's linkage criterion according to which samples or clusters are merged. A graphic visualisation of the samples' classification was presented as a tree diagram, and PCA was visualised in a score scatter plot. Covariance matrices were used as a basis for the calculation of principal components.

\section{Results and discussion}

To realise the aim of this study, DSC scans, FTIR and Raman spectra were recorded for furosemide and caffeine, as well as mixtures of both components and potential cocrystals prepared by liquid-assisted grinding. The formation of co-crystals was confirmed by a comparison of scans or spectra of physical mixtures with those of products obtained by grinding with the addition of acetonitrile, a method which allows differences to be recognised in sample melting points or chemical structures. In the second stage of the work, PCA and CA were used to gain information that can be indirectly acquired from DSC scans, and FTIR and Raman spectra.

\section{Interpretation of DSC scans}

DSC lends itself well to the screening and confirmation of co-crystal formation since a single endothermic event on a DSC scan caused by a sample melting at a different temperature to that of the API or coformer may be sufficient to confirm co-crystal formation. Scans of the samples studied are presented in Fig. 2. Furosemide (Fig. 2h) displays two subsequent endothermic peaks followed by one exothermic (Table 1). The first endothermic event is due to the polymorphic transition of API, while the other is connected with the melting (endothermic peak) or decomposition (exothermic event) of furosemide. The endothermic peak above $260{ }^{\circ} \mathrm{C}$ reflects the melting of the furosemide decomposition product. Polymorphic transition at $\sim 137{ }^{\circ} \mathrm{C}$ and melting with decomposition at $\sim 223{ }^{\circ} \mathrm{C}$ indicate that polymorph form I was used in this study [26, 27]. In the case of caffeine (Fig. 2g), the DSC scan shows only two endothermic effects (Table 1), the first probably being due to the polymorphic transition of API

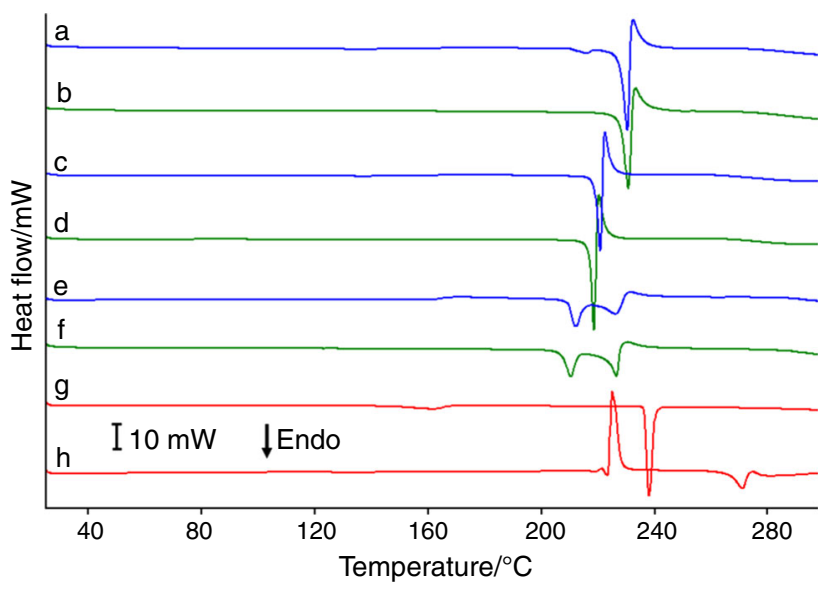

Fig. 2 DSC scans of furosemide-caffeine physical mixtures at drug/coformer ratios: (a) 1:1, (c) 2:1, (e) 1:2, furosemide-caffeine co-crystals at drug/coformer ratios: (b) 1:1, (d) 2:1, (f) 1:2, (g) caffeine, $(h)$ furosemide

and the second can be attributed to the final melting of caffeine. These data in turn indicate that caffeine form II was used in this study [28].

DSC scans of physical mixtures of furosemide with caffeine (Fig. 2, scans a, c, e) display two weak endothermic events at approximately 137 and $160{ }^{\circ} \mathrm{C}$, a probable result of polymorphic transitions of furosemide and caffeine, respectively. Furthermore, for physical mixtures at 1:1 and 2:1 molar ratios, two consecutive endothermic peaks followed by an exothermic event are observed at higher temperatures. As shown in Table 1, enthalpy values for transitions in the fourth peak are tenfold higher than those in the third. The opposite situation is observed for physical mixture at 1:2 molar ratio (Fig. 2e) where the enthalpy value for transition in the third peak is twofold higher than that for the fourth.

DSC scans of potential co-crystals of furosemide with caffeine prepared in different molar ratios are shown in Fig. 2 (scans b, d, f). Co-crystals in molar ratios of 1:1 and 2:1 reveal only a single endothermic effect followed by an exothermic peak, whereas co-crystal at 1:2 molar ratio shows two endothermic events characterised by comparable enthalpy transition values (Table 1). Moreover, weak endothermic events due to polymorphic transitions of both furosemide and caffeine are also observed in the DSC scan of co-crystal at 1:2 molar ratio. The implication is, therefore, that DSC indicated co-crystal formation in 1:1 and 2:1 molar ratios. The melting point of the former co-crystal is consistent with previous studies $[2,26]$.

PCA and CA were used to confirm the findings obtained by DSC. Variations in the data for matrices created in this study, which consist of physical mixtures and co-crystals of API and coformer, are listed in Table 2. PC1 and PC2 taken together explained more than $70 \%$ of the total 
Table 1 Temperatures of onset $\left(T_{\text {onset }}\right)$ and peak $\left(T_{\text {peak }}\right)$ and the enthalpy of transitions $(\Delta H)$ obtained from DSC scans of furosemide, caffeine, their physical mixtures and potential co-crystals

\begin{tabular}{|c|c|c|c|c|c|}
\hline Samples & Molar ratios & Peaks number & $T_{\text {onset }} /{ }^{\circ} \mathrm{C}$ & $T_{\text {peak }} /{ }^{\circ} \mathrm{C}$ & $\Delta H / \mathrm{J} \mathrm{g}^{-1}$ \\
\hline \multirow[t]{4}{*}{ Furosemide } & & 1 & 129.38 & 137.16 endo $(w)$ & 4.63 \\
\hline & & 2 & 221.20 & 223.28 endo $(w)$ & 4.19 \\
\hline & & 3 & 223.59 & 226.22 exo (s) & 107.49 \\
\hline & & 4 & 266.25 & 270.53 endo $(\mathrm{m})$ & 42.85 \\
\hline \multirow[t]{2}{*}{ Caffeine } & & 1 & 147.16 & 161.11 endo $(w)$ & 16.45 \\
\hline & & 2 & 235.99 & 236.24 endo (s) & 104.22 \\
\hline \multirow[t]{15}{*}{ Physical mixtures } & $1: 1$ & 1 & 128.68 & 136.46 endo $(w)$ & 1.75 \\
\hline & & 2 & 149.80 & 159.46 endo $(w)$ & 2.75 \\
\hline & & 3 & 211.53 & 215.05 endo $(w)$ & 5.91 \\
\hline & & 4 & 227.37 & 228.71 endo $(\mathrm{m})$ & 50.18 \\
\hline & & 5 & 229.47 & 232.80 exo $(\mathrm{m})$ & 45.33 \\
\hline & $2: 1$ & 1 & 128.21 & 136.79 endo $(w)$ & 2.38 \\
\hline & & 2 & 148.65 & 159.63 endo $(w)$ & 1.62 \\
\hline & & 3 & 210.47 & 213.24 endo $(w)$ & 3.56 \\
\hline & & 4 & 218.67 & 219.70 endo $(\mathrm{m})$ & 47.75 \\
\hline & & 5 & 222.63 & 224.18 exo $(\mathrm{m})$ & 39.44 \\
\hline & $1: 2$ & 1 & 129.38 & 136.98 endo $(w)$ & 0.75 \\
\hline & & 2 & 146.58 & 160.32 endo $(w)$ & 3.68 \\
\hline & & 3 & 209.22 & 211.53 endo $(\mathrm{m})$ & 41.95 \\
\hline & & 4 & 220.99 & 225.57 endo $(\mathrm{m})$ & 19.15 \\
\hline & & 5 & 229.23 & 231.11 exo $(w)$ & 3.68 \\
\hline \multirow[t]{9}{*}{ Co-crystals } & $1: 1$ & 1 & 225.06 & 227.07 endo (s) & 96.63 \\
\hline & & 2 & 231.88 & 233.50 exo $(w)$ & 11.63 \\
\hline & $2: 1$ & 1 & 216.24 & 216.94 endo $(w)$ & 11.62 \\
\hline & & 2 & 220.01 & 220.89 exo $(\mathrm{m})$ & 57.71 \\
\hline & $1: 2$ & 1 & 126.57 & 129.46 endo $(w)$ & 0.38 \\
\hline & & 2 & 153.12 & 158.78 endo $(w)$ & 0.74 \\
\hline & & 3 & 207.08 & 209.65 endo $(\mathrm{m})$ & 43.71 \\
\hline & & 4 & 222.54 & 225.81 endo $(w)$ & 9.34 \\
\hline & & 5 & 227.78 & 230.23 exo $(\mathrm{m})$ & 21.02 \\
\hline
\end{tabular}

Intensity of the DSC peaks: $w$ weak, $m$ medium, $s$ strong

variance, meaning that the results of PCA can be visualised in a two-dimensional score scatter plot. The localisation of physical mixture and co-crystal prepared in the same molar ratio and on the same PCA plane along the PC1, PC2 or PC3 axes indicates that no process of co-crystallisation occurred and that consequently there are no differences in the thermal properties of the samples. By contrast, the localisation of physical mixture and co-crystal prepared in the same molar ratio in the opposite PCA space along PC1, PC2 or PC 3 axes does reveal co-crystal formation.

Figure 3 presents the score plots for the first three PCs for furosemide and caffeine physical mixtures and their potential co-crystals based on heat flow value. As can be seen, physical mixture and co-crystal in a 1:2 molar ratio (samples e and f) are grouped in the same space as PC1,
PC2 and PC3 (Fig. 3a, b). It bears a significant similarity between physical mixture and co-crystal (both in 1:2 molar ratio). Furosemide-caffeine co-crystals in $1: 1$ and 2:1 molar ratios (samples b, d) form a cluster in the central part of the PCA score plots while their physical mixtures are separate from the co-crystals cluster. PC1 distinguishes between physical mixture in a 1:1 molar ratio and cocrystal cluster. PC2 correlates positively with physical mixture in a 2:1 molar ratio but negatively with the cocrystals cluster. Moreover, PC3 with $17.09 \%$ of explained variation mainly distinguishes co-crystal in 1:1 molar ratio from others samples.

The evident clustering tendency of samples is depicted in Fig. 4 in the form of a CA tree diagram. The first cluster is formed below $33 \%$ of the maximum distance by 
Table 2 Eigenvalues and variation in the data used for PCA calculations for physical mixtures and co-crystals of furosemide with caffeine

\begin{tabular}{llcll}
\hline Techniques & $\begin{array}{l}\text { Principal } \\
\text { components }\end{array}$ & Eigenvalue & $\begin{array}{l}\text { Variance/ } \\
\%\end{array}$ & $\begin{array}{l}\text { Cumulative } \\
\text { variance/\% }\end{array}$ \\
\hline DSC & PC1 & 6.71 & 45.99 & 45.99 \\
FTIR & & 4.30 & 58.16 & 58.16 \\
Raman & & 14.79 & 79.39 & 79.39 \\
DSC & PC2 & 3.86 & 26.48 & $72.47^{\mathrm{a}}$ \\
FTIR & & 2.54 & 34.39 & $92.55^{\mathrm{a}}$ \\
Raman & & 3.11 & 16.72 & $96.11^{\mathrm{a}}$ \\
DSC & PC3 & 2.49 & 17.09 & $89.56^{\mathrm{b}}$ \\
FTIR & & 0.30 & 4.00 & $96.55^{\mathrm{b}}$ \\
Raman & & 0.64 & 3.43 & $99.54^{\mathrm{b}}$
\end{tabular}

${ }^{\mathrm{a}} \mathrm{PC} 1$ and $\mathrm{PC} 2$ taken together

b PC1, PC2 and PC3 taken together
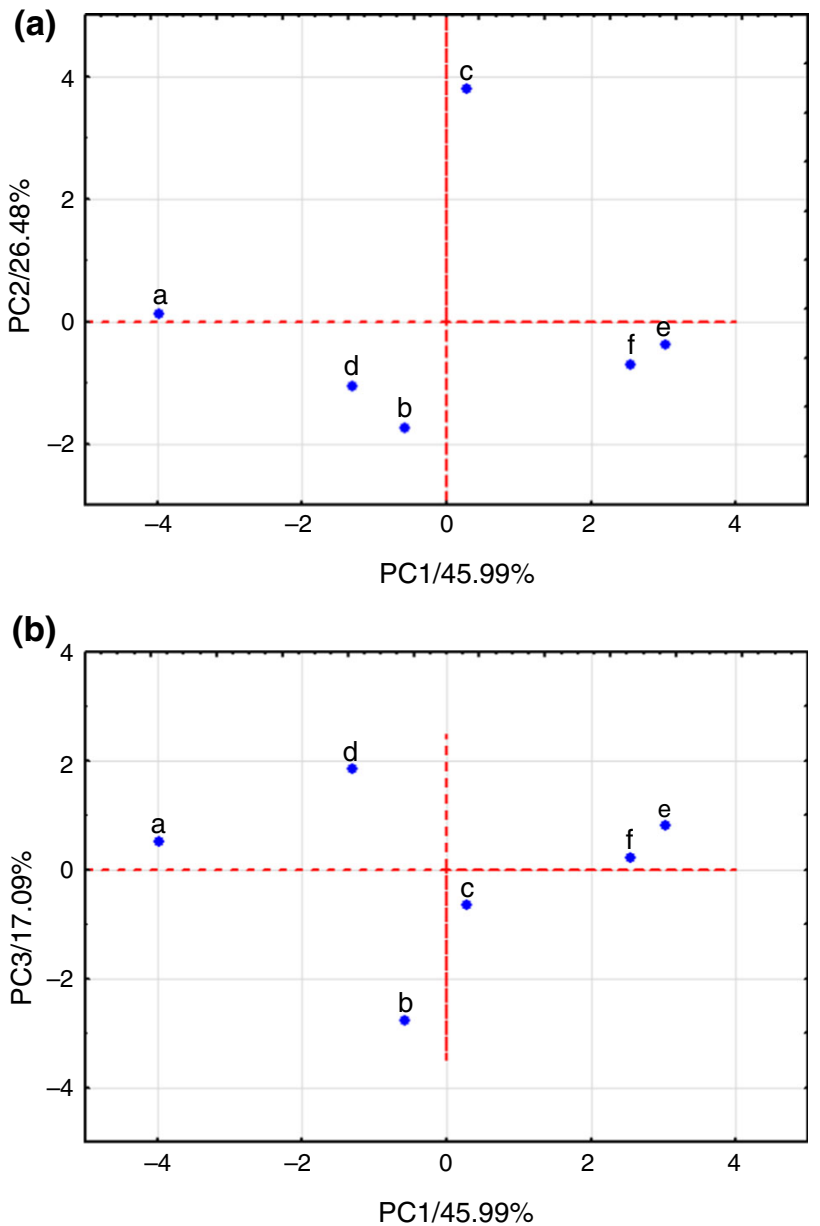

Fig. 3 PCA score plots for: a PC1 and PC2, b PC1 and PC3, calculated for the data acquired from DSC scans. Furosemidecaffeine physical mixtures at drug/coformer ratios: (a) $1: 1$, (c) 2:1,

(e) 1:2, furosemide-caffeine co-crystals at drug/coformer ratios:

(b) $1: 1,(d) 2: 1,(f) 1: 2$

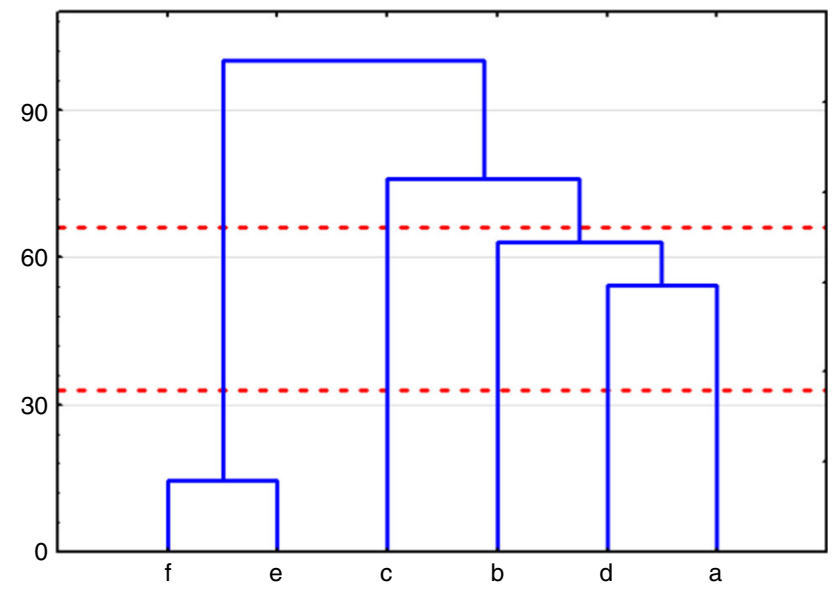

Fig. 4 CA tree diagram for the data obtained from DSC scans. Furosemide-caffeine physical mixtures at drug/coformer ratios: (a) $1: 1$, (c) $2: 1$, (e) 1:2; furosemide-caffeine co-crystals at drug/coformer ratios: (b) 1:1, (d) 2:1, (f) 1:2

potential co-crystal and physical mixture in 1:2 molar ratio (samples e, f). Due to the revealed similarity between samples, it could be assumed that process of cocrystallisation did not occur. On the other hand, a large cluster consisting of the remaining samples is formed above $33 \%$ of the maximum distance. The distribution of physical mixtures and co-crystals prepared in 1:1 and 2:1 molar ratios in different clusters reveals co-crystal formation.

These results show that PCA and CA confirm cocrystal formation by identifying co-crystals in 1:1 and 2:1 molar ratios from among other samples. However, the PC1 distinguish physical mixture in 1:1 molar ratio from co-crystal in the same molar ratio more precisely than is the case for co-crystal and physical mixture in 2:1 molar ratio, PC1 in particular does not differentiate samples in 2:1 molar ratio. This may indicate that the co-crystallisation process could not occur for components mixed in 2:1 molar ratio.

\section{Interpretation of FTIR spectra}

FTIR spectroscopy is used to confirm co-crystal formation because of its ability to detect differences in the chemical structures of samples indicative of the formation of hydrogen bonding. The spectra of furosemide, caffeine, their physical mixtures and potential co-crystals in molar ratios 1:1, 2:1 and 1:2 are presented in Fig. 5. Characteristic bands for furosemide (spectrum $\mathrm{g}$ ) are found at 3399.4 and $3350.3 \mathrm{~cm}^{-1}\left(\mathrm{NH}_{2}\right.$ stretching vibration), $3284.2 \mathrm{~cm}^{-1}$ $(\mathrm{N}-\mathrm{H} \quad$ stretching $), \quad 1672.0 \mathrm{~cm}^{-1} \quad(\mathrm{C}=\mathrm{O} \quad$ stretching $)$, $1591.8 \mathrm{~cm}^{-1}$ (N-H bending), $1322.5 \mathrm{~cm}^{-1}\left(\mathrm{R}_{-} \mathrm{SO}_{2}\right.$ symmetric stretching), $1142.1 \mathrm{~cm}^{-1}$ (S-O stretching) and $744.6 \mathrm{~cm}^{-1}$ (C-Cl stretching). Caffeine (spectrum h) 


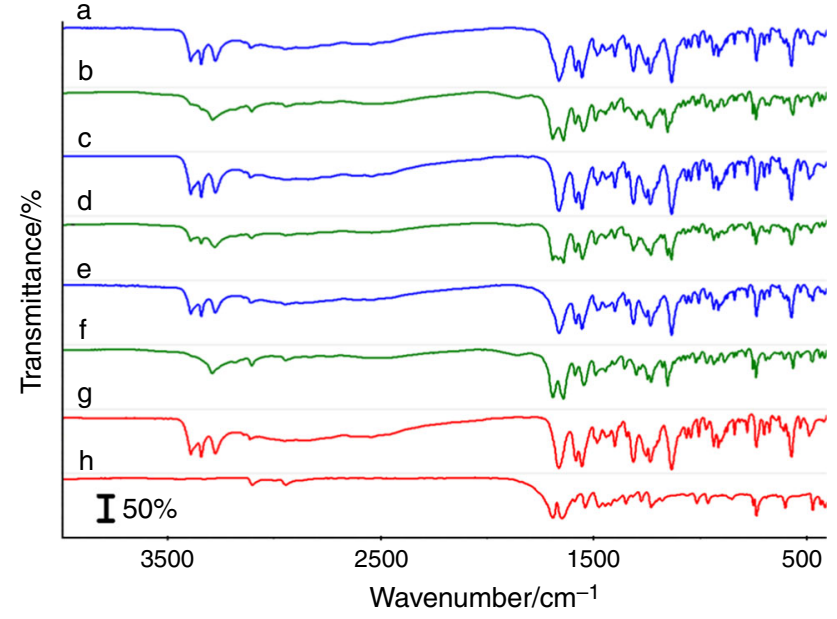

Fig. 5 FTIR spectra of furosemide-caffeine physical mixtures at drug/coformer ratios: (a) 1:1, (c) 2:1, (e) 1:2, furosemide-caffeine cocrystals at drug/coformer ratios: (b) 1:1, (d) 2:1, (f) 1:2, $(g)$ furosemide, $(h)$ caffeine

shows characteristic bands at $1700.7 \mathrm{~cm}^{-1}(\mathrm{C}=\mathrm{O}$ in-phase stretching), $1655.3 \mathrm{~cm}^{-1}$ ( $\mathrm{C}=\mathrm{O}$ out-of-phase stretching), $1598.1 \mathrm{~cm}^{-1} \quad(\mathrm{C}=\mathrm{C}$ and $\mathrm{C}=\mathrm{N}$ stretching $), 1548.2 \mathrm{~cm}^{-1}$ (imidazole ring stretching). These data are compatible with those found in the literature [2, 28, 29].

As shown in Fig. 5, the FTIR spectra for physical mixtures of furosemide with caffeine (spectra a, c, e) displayed bands specific for both components and no significant shifts in bands wavenumbers were detected. However, differences in transmittance intensity were observed, probably due to the variety in molar composition of the physical mixtures. On the other hand, the spectrum of cocrystal in 1:1 molar ratio (Fig. 5b) revealed a shift in the characteristic bands of API and coformer to lower or higher wavenumbers. Bands of furosemide at 1591.8 and $1142.1 \mathrm{~cm}^{-1}$ shifted to 1595.0 and $1161.6 \mathrm{~cm}^{-1}$, respectively, in the co-crystal spectrum, while band of caffeine at $1655.3 \mathrm{~cm}^{-1}$ shifted to $1650.2 \mathrm{~cm}^{-1}$. Moreover, the disappearance of characteristic bands of furosemide (3399.4, 3350.3 and $3284.2 \mathrm{~cm}^{-1}$ ) and the appearance of a new band at $3297.6 \mathrm{~cm}^{-1}$ were also detected in the co-crystal spectrum. Similar bands were also found in the FTIR spectrum of co-crystal in 1:2 molar ratio (Fig. 5f). Shifts in the spectral position of bands assigned for amino, carbonyl and sulfonyl groups of furosemide and the carbonyl group of caffeine confirm the formation of hydrogen bonds between free hydrogen donors, and acceptors of both components confirm co-crystal formation [10]. On the other hand, the similarity of the physical mixture and cocrystal spectra (both in 2:1 molar ratio) suggests that cocrystallisation did not occur (Fig. 5, spectra c, d).

As in the case of DSC, the data acquired from FTIR spectra were interpreted using unsupervised chemometric methods, PCA and CA. The PCA score plots for the studied samples are shown in Fig. 6. Detailed inspection of Fig. 6a reveals that physical mixtures create a cluster on the righthand side of the plot, whereas co-crystals are grouped to the left. This shows that PC1 (which accounts for 58.16\% of the total variance) primarily distinguishes co-crystals from physical mixtures. However, a potential co-crystal in molar ratio 2:1 (sample d) is located in the central position of the plot, in closer proximity to physical mixtures than to other co-crystals. This may be due to the spectral similarities between co-crystal and physical mixture in 2:1 molar ratio and may exclude co-crystal formation in 2:1 molar ratio. The distribution of samples along PC2 (Fig. 6a) and PC3 (Fig. 6b) reveals that co-crystals in molar ratios 1:1 and 1:2 are clearly distinct. These co-crystals correlated with PC2 and PC3, negatively (co-crystal 1:1) and positively (co-crystal 1:2).
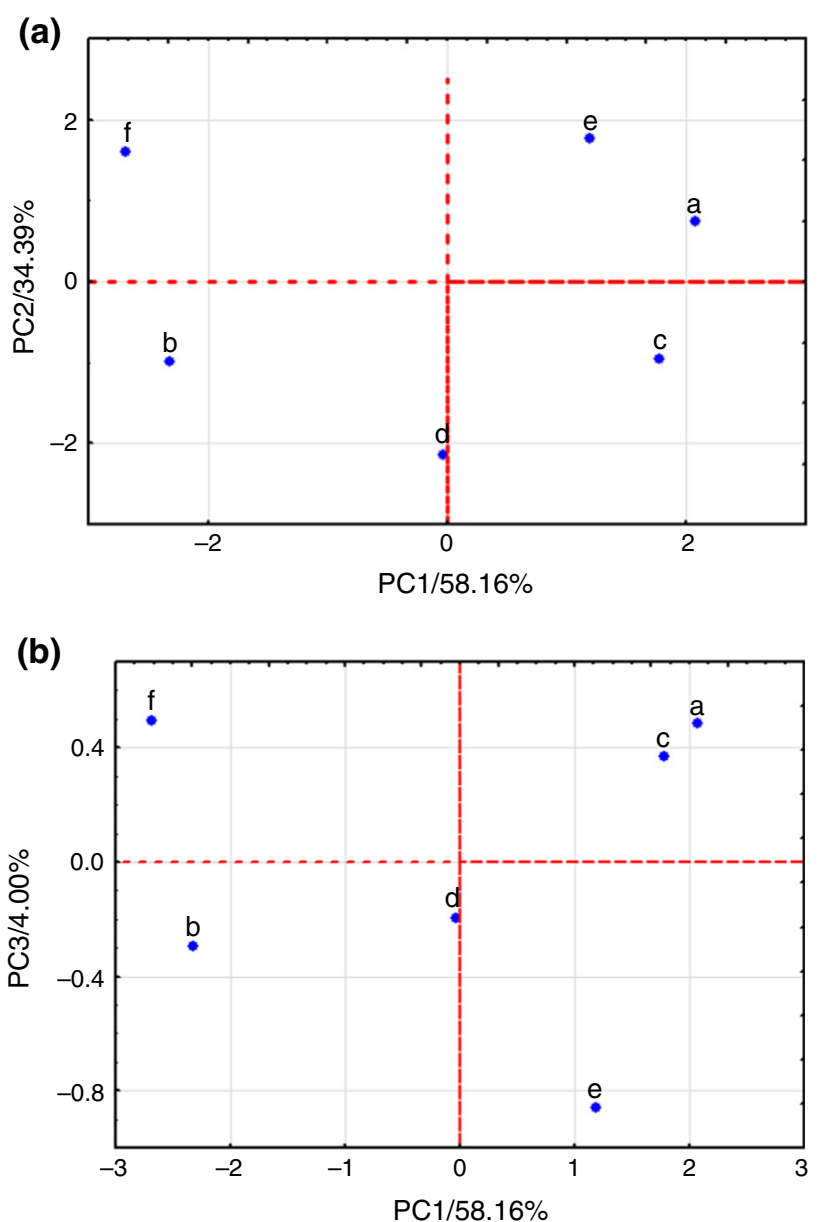

Fig. 6 PCA score plots for: a PC1 and PC2, b PC1 and PC3, calculated for the data acquired from FTIR spectra. Furosemidecaffeine physical mixtures at drug/coformer ratios: (a) 1:1, (c) 2:1, (e) 1:2, furosemide-caffeine co-crystals at drug/coformer ratios: (b) $1: 1,(d) 2: 1,(f) 1: 2$ 


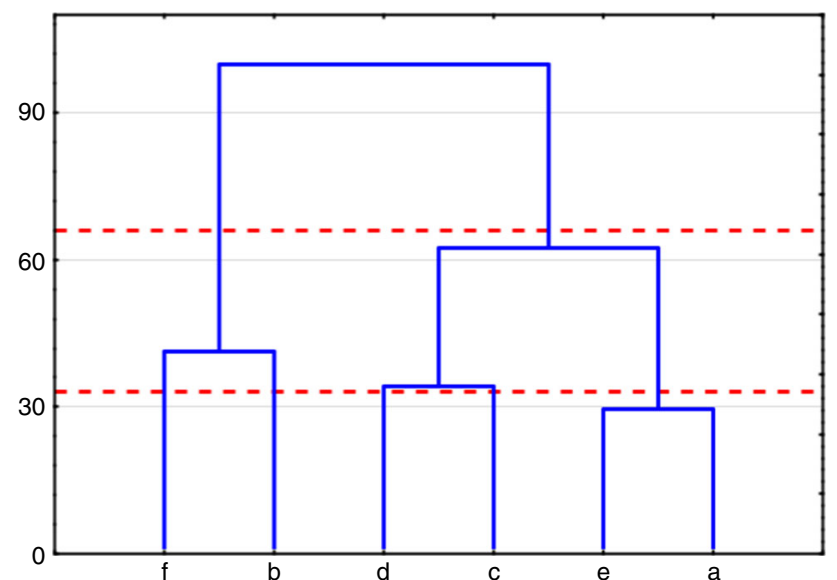

Fig. 7 CA tree diagram for the data obtained from FTIR spectra. Furosemide-caffeine physical mixtures at drug/coformer ratios: (a) $1: 1$, (c) $2: 1$, (e) 1:2, furosemide-caffeine co-crystals at drug/coformer ratios: (b) 1:1, (d) 2:1, (f) 1:2

As shown in Fig. 7, CA distributes the physical mixtures and co-crystals under study into two clusters situated below $66 \%$ of the maximum distance. Co-crystals in 1:1 and 1:2 molar ratios (samples b, f) are grouped in the first cluster at approximately $40 \%$ of the maximum distance, which confirms co-crystallisation. The second cluster splits into two subsequent clusters which group physical mixture and co-crystal in 2:1 molar ratio (samples c, d) and physical mixtures in 1:1 and 1:2 molar ratios (samples a, e), respectively. Both clusters created at approximately $30 \%$ of the maximum distance revealed that the co-crystal in a 2:1 molar ratio has not been prepared using liquid-assisted grinding.

\section{Interpretation of Raman spectra}

Raman and FTIR spectroscopies are complementary tools because other molecules and bonding are active in their spectra. Raman spectra of physical mixtures, co-crystals and both substances alone are shown in Fig. 8. Characteristic bands of furosemide (spectrum g) occur at $1593.5 \mathrm{~cm}^{-1}\left(\mathrm{NH}_{2}\right.$ scissoring vibration), $1503.1 \mathrm{~cm}^{-1}$ (aromatic ring stretching), $1334.2 \mathrm{~cm}^{-1}\left(\mathrm{SO}_{2}\right.$ asymmetric stretching), $1213.5 \mathrm{~cm}^{-1}(\mathrm{O}-\mathrm{H}$ bending in-plane, $\mathrm{C}-\mathrm{O}$ stretching), $1144.6 \mathrm{~cm}^{-1}\left(\mathrm{SO}_{2}\right.$ symmetric stretching), $681.8 \mathrm{~cm}^{-1}$ (C-Cl stretching), $577.6 \mathrm{~cm}^{-1}(\mathrm{O}-\mathrm{H}$ bending out-of-plane). Caffeine (spectrum h) displays specific bands at $1692.6 \mathrm{~cm}^{-1} \quad(\mathrm{C}=\mathrm{O}$ stretching in-phase $)$, $1649.9 \mathrm{~cm}^{-1}$ (C=O stretching out-of-phase), $1594.5 \mathrm{~cm}^{-1}$ $(\mathrm{C}=\mathrm{C}$ symmetric stretching, $\mathrm{C}-\mathrm{N}$ symmetric stretching, $\mathrm{CH}_{3}$ symmetric bending), $1322.7 \mathrm{~cm}^{-1}$ (imidazole trigonal ring stretching), $\quad 1065.4 \mathrm{~cm}^{-1} \quad(\mathrm{H}-\mathrm{C}=\mathrm{N}$ bending $)$, $549.3 \mathrm{~cm}^{-1}$ ( $\mathrm{CH}$ bending, $\mathrm{CH}_{3}$ bending, $\mathrm{CH}_{3}$ rocking).

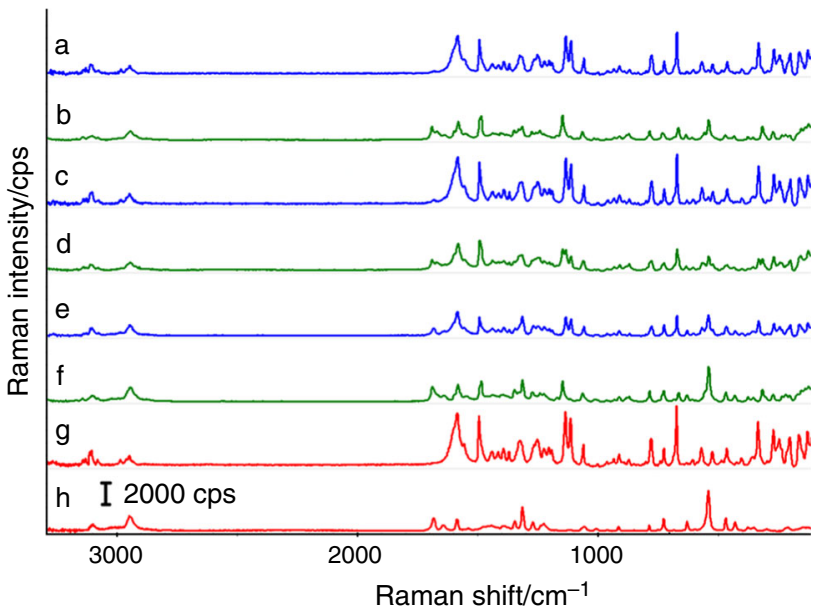

Fig. 8 Raman spectra of furosemide-caffeine physical mixtures at drug/coformer ratios: (a) 1:1, (c) 2:1, (e) 1:2, furosemide-caffeine cocrystals at drug/coformer ratios: (b) 1:1, (d) 2:1, (f) 1:2, $(g)$ furosemide, $(h)$ caffeine

These characteristic bands are consistent with those found in the literature for furosemide [29] and caffeine [28, 30], respectively. Although all characteristic bands for both components are also found in the Raman spectra of physical mixtures (spectra a, c, e), differences in Raman intensity values were detected between samples due to the differences in molar ratio of furosemide and caffeine. Additionally, a shifting of characteristic bands to API and coformer occurs in co-crystals (spectra b, d, f). For instance, bands of furosemide at 1593.5 and $1334.2 \mathrm{~cm}^{-1}$ were shifted in the co-crystal spectrum, to $\sim 1590$ and $\sim 1325 \mathrm{~cm}^{-1}$, respectively, whereas band of caffeine at $1692.6 \mathrm{~cm}^{-1}$ was shifted to $\sim 1699 \mathrm{~cm}^{-1}$. Moreover, the Raman spectra of co-crystals in molar ratios 1:1 and 1:2 bear distinct similarities, but are only slightly similar to the spectrum of co-crystal in 2:1 molar ratio. In this case, insignificant shifts in the spectral position of bands are observed for co-crystal in 2:1 molar ratio as compared with spectra of the others.

The PCA score plots for the first three principal components calculated for the data acquired from Raman spectra are presented in Fig. 9. To emphasise the importance of Raman spectroscopy in the detection of co-crystals, it is important to mention that in this case PC1 explains a higher percentage of the total variance than that obtained for the data acquired from DSC or FTIR (Table 2). Thus, the distribution of samples along the PC1 axis is of great importance. As shown in Fig. 9a, the scatter of physical mixtures and co-crystals is similar to that obtained in the case of FTIR (Fig. 6). The difference lies in the fact that co-crystals in 1:1 and 1:2 molar ratios create a separate cluster on the right of the plot, whereas all physical mixtures and potential co-crystals in a 2:1 molar ratio 

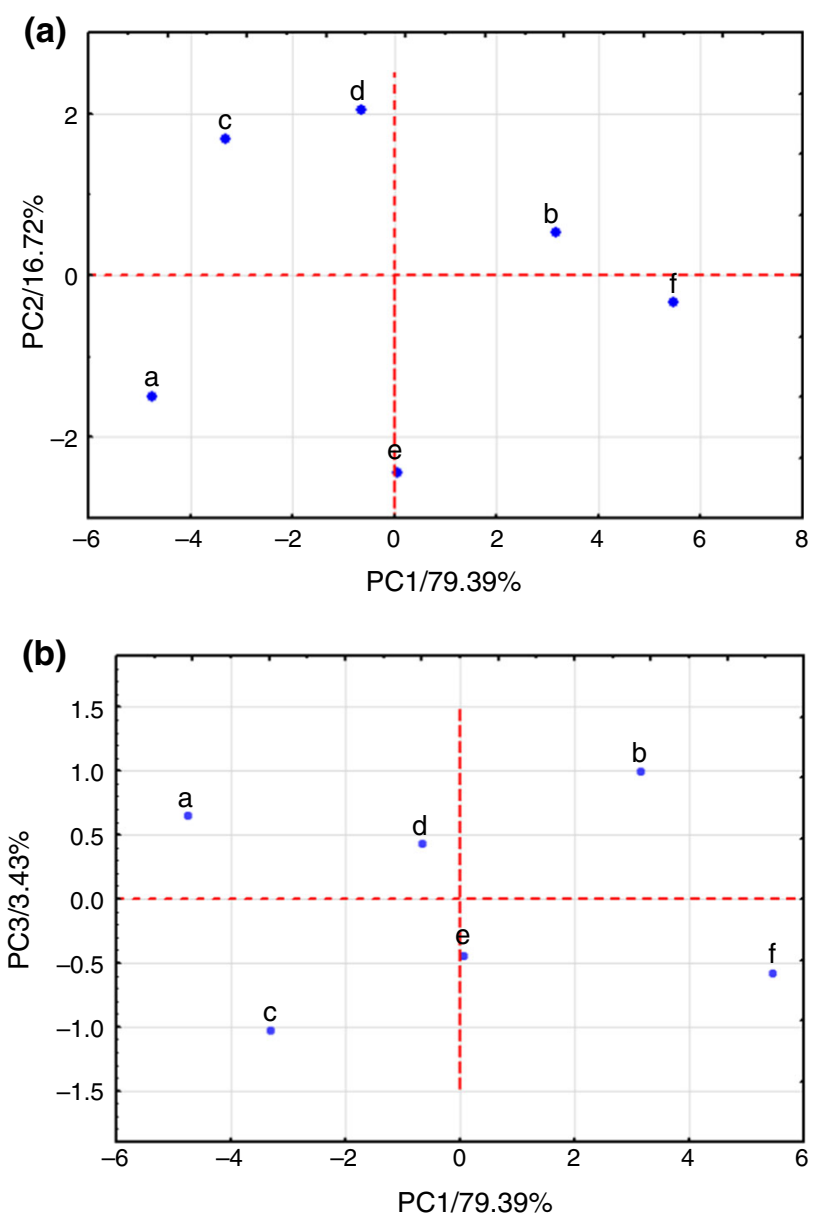

Fig. 9 PCA score plots for: a PC1 and PC2, b PC1 and PC3, calculated for the data acquired from Raman spectra. Furosemidecaffeine physical mixtures at drug/coformer ratios: (a) 1:1, (c) 2:1, (e) 1:2, furosemide-caffeine co-crystals at drug/coformer ratios: (b) 1:1, (d) 2:1, (f) 1:2

are grouped to the left. In other words, PC1 clearly determines two co-crystals (samples $b$, f), and physical mixtures (samples a, c, d, e) confirm that no co-crystal in a 2:1 molar ratio exists, a finding further corroborated by the PCA score plot of PC1 and PC3 (Fig. 9b).

The tree diagram calculated using the data acquired from Raman spectra is shown in Fig. 10. It is similar to that calculated for the FTIR spectra, with the only differences being in the proximity distances between CA trees. The cluster grouping of co-crystals in 1:1 and 1:2 molar ratios is created in the lowest percentage of the maximum distance (below 33\%). This confirms that both co-crystals have similar Raman spectra and that the clustering tendency is stronger in comparison with other clusters.

In conclusion, the findings obtained by PCA and CA for the data acquired from DSC scans differ from those created for the data obtained from FTIR and Raman spectra (Table 3). However, regardless of which matrix was the starting point for calculations, the formation of co-crystal

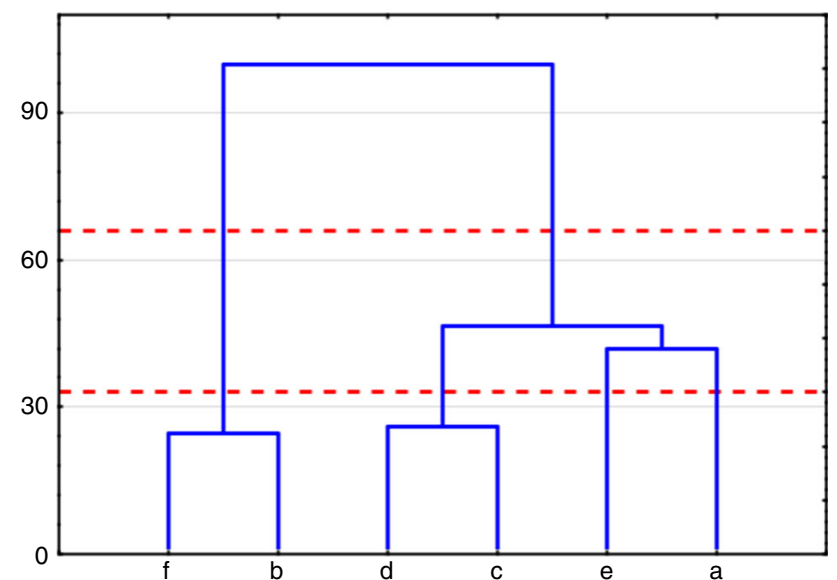

Fig. 10 CA tree diagram for the data obtained from Raman spectra. Furosemide-caffeine physical mixtures at drug/coformer ratios: (a) $1: 1,(c)$ 2:1, (e) 1:2, furosemide-caffeine co-crystals at drug/coformer ratios: $(b)$ 1:1, (d) 2:1, (f) 1:2

Table 3 Results obtained by using multivariate statistical techniques as supplementary tools for interpretation of the DSC, FTIR and Raman data for physical mixtures and co-crystals of furosemide with caffeine

\begin{tabular}{llll}
\hline Techniques & Molar ratio & PCA & CA \\
\hline DSC & $1: 1$ & + & + \\
& $2: 1$ & + & + \\
& $1: 2$ & - & - \\
FTIR & $1: 1$ & + & + \\
& $2: 1$ & - & - \\
\multirow{2}{*}{ Raman } & $1: 2$ & + & + \\
& $1: 1$ & + & + \\
& $2: 1$ & - & - \\
& $1: 2$ & + & + \\
\hline
\end{tabular}

+ , Co-crystal; -, physical mixture

in 1:1 molar ratio was confirmed in each case. Furthermore, co-crystals in molar ratios 2:1 (DSC data) and 1:2 (FTIR and Raman data) were also detected in this study. To explain the discrepancy in chemometric analysis of the data, it is necessary to consider which characteristic features of a sample under study are being measured. DSC shows that phase transitions, such as polymorphic transitions, melting and crystallisation, occurred in a sample during the heating process, while FTIR and Raman reveal the chemical structure of a sample reflected by characteristic vibrational bands. Hence, a comparison of the melting points of co-crystal and API or coformer indicates cocrystal formation. However, it should also be borne in mind that changes in melting point can also be the result of the formation of a eutectic mixture. On the other hand, it has been established that API and coformer interact via nonionic and non-covalent intermolecular interactions, such as 
van der Waals forces, $\pi-\pi$ interactions, and most importantly, hydrogen bonding [10]. Thus, changes in the spectral position of bands characteristic to API or coformer indicate co-crystal formation. Taking all of the above into consideration, it would appear that spectroscopic methods which reflect the chemical bonding of substances provide more reliable information on co-crystal formation than the DSC method, which illustrates phase transitions. In this situation, PCA and CA examination of the data acquired from FTIR and Raman spectra could be more reliable than that data received from DSC scans.

As reported in the literature, co-crystal formation of furosemide with caffeine was induced by the polymorphic transition of API at $137{ }^{\circ} \mathrm{C}$ during the heating of physical mixture. Moreover, co-crystal in 1:1 molar ratio formed a eutectic mixture with excess of caffeine, whereas no such mixture was formed by excess of furosemide [26]. In our study, a similar effect to that reported in the literature was observed in the case of DSC scans of physical mixture in 1:2 molar ratio and possible co-crystal in the same molar ratio. PCA and CA examination of the values of heat flow is a stronger indicator of the similarities between potential co-crystal and physical mixture in the same molar ratio (1:2) than data from FTIR and Raman spectra. This study suggests that the complex analysis of the data obtained by chemometric examination of DSC scans as well as FTIR and Raman spectra enables distinct separation of co-crystal from a eutectics mixture consisting of co-crystal and caffeine. For this reason, it was concluded that co-crystals of furosemide and caffeine were obtained only in 1:1 molar ratio, while co-crystals in molar ratio $2: 1$ and 1:2 were not formed.

\section{Conclusions}

The results of a DSC, FTIR and Raman study of furosemide, caffeine, physical mixtures and potential cocrystals of both components in molar ratios 1:1, 2:1 and 1:2 supported by two unsupervised pattern recognition methods (PCA and CA) revealed significant discrepancies between the data obtained. In each case (DSC, FTIR and Raman study), co-crystal in 1:1 molar ratio was detected, while co-crystals in molar ratios 2:1 and 1:2 were also confirmed by DSC, and FTIR and Raman, respectively. These discrepancies are related to specific features of the samples being measured. As a result of this study, it was established that spectroscopic methods which reflect the chemical bonding of substances provide more reliable information on co-crystal formation than a DSC method that illustrates phase transitions. In this situation, PCA and CA examination of the data acquired from FTIR and Raman spectra could be more reliable than that of data received from DSC scans. Finally, co-crystal formation could only be confirmed where the results obtained from PCA and CA examination of the data acquired from DSC, FTIR and Raman methods are compatible.

Acknowledgements The investigations were financially supported by a statutory research, Grant No. ST-15, from the Ministry of Science and Higher Education, Poland.

Open Access This article is distributed under the terms of the Creative Commons Attribution 4.0 International License (http://crea tivecommons.org/licenses/by/4.0/), which permits unrestricted use, distribution, and reproduction in any medium, provided you give appropriate credit to the original author(s) and the source, provide a link to the Creative Commons license, and indicate if changes were made.

\section{References}

1. Blagden N, de Matas M, Gavan PT, York P. Crystal engineering of active pharmaceutical ingredients to improve solubility and dissolution rates. Adv Drug Deliv Rev. 2007;59:617-30.

2. Goud NR, Gangavaram S, Suresh K, Pal S, Manjunatha SG, Nambiar S, Nangia A. Novel furosemide cocrystals and selection of high solubility drugs forms. J Pharm Sci. 2012;101:664-80.

3. Zhang G-C, Lin H-L, Lin S-Y. Thermal analysis and FTIR spectral curve-fitting investigation of formation mechanism and stability of indomethacin-saccharin cocrystals via solid-state grinding process. J Pharm Biomed Anal. 2012;66:162-9.

4. Basavoju S, Boström D, Velaga SP. Indomethacin-saccharin cocrystal: design, synthesis and preliminary pharmaceutical characterization. Pharm Res. 2008;25:530-41.

5. FDA. Guidance for industry. Regulatory classification of pharmaceutical co-crystals (2013). http://www.fda.gov/downloads/ Drugs/Guidances/UCM281764.pdf. Accessed 30 Oct 2016.

6. Thakuria R, Delori A, Jones W, Lipert MP, Roy L, RodríguezHornerdo N. Pharmaceutical cocrystals and poorly soluble drugs. Int J Pharm. 2013;453:101-25.

7. Patel PV, Brahmbhatt H, Upadhyay UM, Shah V. A review on increased therapeutic efficiency of drugs by pharmaceutical cocrystal approach. Int J Pharm Sci Rev Res. 2012;16:140-8.

8. Schultheiss N, Newman A. Pharmaceutical cocrystals and their physicochemical properties. Cryst Growth Des. 2009;9:2950-67.

9. Qiao N, Li M, Schlindwein W, Malek N, Davies A, Trappitt G. Pharmaceutical cocrystals: an overview. Int $\mathrm{J}$ Pharm. 2011;419:1-11.

10. Rehder S, Klukkert M, Löbmann KAM, Strachan CJ, Sakmann A, Gordon K, Rades T, Leopold CS. Investigation of the formation process of two piracetam cocrystals during grinding. Pharmaceutics. 2011;3:706-22.

11. Lin H-L, Zhang G-C, Lin S-Y. Real-time co-crystal screening and formation between indomethacin and saccharin via DSC analytical technique or DSC-FTIR microspectroscopy. J Therm Anal Calorim. 2015;120:679-87.

12. Lin H-L, Zhang G-C, Hsu P-C, Lin S-Y. A portable fiber-optic Raman analyzer for fast real-time screening and identifying cocrystal formation of drug-coformer via grinding process. Microchem J. 2013;110:15-20.

13. Sarraguça MC, Paisana M, Pinto J, Lopes JA. Real-time monitoring of cocrystallization processes by solvent evaporation: a near infrared study. Eur J Pharm Sci. 2016;90:76-84.

14. Stoler E, Warner JC. Non-covalent derivatives: cocrystals and eutectics. Molecules. 2015;20:14833-48. 
15. Craig DQM, Reading M, editors. Thermal analysis of pharmaceuticals. Boca Raton: CRC Press; 2007.

16. Šašić S, Ozaki Y, editors. Raman, infrared and near-infrared chemical imaging. Hoboken: Wiley; 2010.

17. Miller JN, Miller JC. Statistics and chemometrics for analytical chemistry. 6th ed. Essex: Pearson Education; 2010.

18. Massart DL, Kaufman L. The interpretation of analytical chemical data by the use of cluster analysis. New York: Wiley; 1983.

19. Rojek B, Wesolowski M. Compatibility studies of hydrocortisone with excipients using thermogravimetric analysis supported by multivariate statistical analysis. J Therm Anal Calorim. 2017; 127:543-53.

20. Rojek B, Wesolowski M. Fourier transform infrared spectroscopy supported by multivariate statistics in compatibility study of atenolol with excipients. Vib Spectrosc. 2016;86:190-7.

21. Caliandro R, Di Profio G, Nicolotti O. Multivariate analysis of quaternary carbamazepine-saccharin mixtures by X-ray diffraction and infrared spectroscopy. J Pharm Biomed Anal. 2013;78-79:269-79.

22. Soares FLF, Carneiro RL. Evaluation of analytical tools and multivariate methods for quantification of co-former crystals in ibuprofen-nicotinamide co-crystals. J Pharm Biomed Anal. 2014;89:166-75.

23. Sarraguça MC, Ribeiro PRS, Santos AO, Silva MCD, Lopes JA. A PAT approach for the on-line monitoring of pharmaceutical cocrystals formation with near infrared spectroscopy. Int J Pharm. 2014;471:478-84.
24. Sarraguça MC, Ribeiro PRS, Dos Santos AO, Lopes JA. Batch statistical process monitoring approach to a cocrystallization process. J Pharm Sci. 2015;104:4099-108.

25. Karashima M, Kimoto K, Yamamoto K, Kojima T, Ikeda Y. A novel solubilization technique for poorly soluble drugs through the integration of nanocrystal and cocrystal technologies. Eur J Pharm Biopharm. 2016;107:142-50.

26. Pal S, Roopa BN, Abu K, Manjunath SG, Nambiar S. Thermal studies of furosemide-caffeine binary system that forms a cocrystal. J Therm Anal Calorim. 2014;115:2261-8.

27. da Silva RDC, Semaan FS, Novák C, Cavalheiro ETG. Thermal behavior of furosemide. $\mathrm{J}$ Therm Anal Calorim. 2013;111:1933-7.

28. Mukaida M, Watanabe Y, Sugano K, Terada K. Identification and physicochemical characterization of caffeine-citric acid co-crystal polymorphs. Pharm Sci. 2015;79:61-6.

29. Ali H, Singh SK, Verma PRP. Preformulation and physicochemical interaction study of furosemide with different solid lipids. J Pharm Investig. 2015;45:385-98.

30. Pavel I, Szeghalmi A, Moigno D, Cîntă S, Kiefer W. Theoretical and $\mathrm{pH}$ dependent surface enhanced Raman spectroscopy study on caffeine. Biopolymers. 2003;72:25-37. 